\title{
ASYMPTOTIC FORMULAS FOR THE GAMMA FUNCTION BY GOSPER
}

\author{
LONG LiN AND CHAO-PING CHEN
}

Abstract. The main aim of this paper is to give two general asymptotic expansions for the gamma function, which include the Gosper formula as their special cases. Furthermore, we present an inequality for the gamma function.

Mathematics subject classification (2010): Primary 33B15; Secondary 41A60.

Keywords and phrases: Gamma function, asymptotic formula, Bernoulli numbers, fundamental theorem of algebra, Newton's formulas.

\section{REFERENCES}

[1] M. Abramowitz And I. A. Stegun (Editors), Handbook of Mathematical Functions with Formulas, Graphs, and Mathematical Tables, Applied Mathematics Series 55, Ninth printing, National Bureau of Standards, Washington, D. C., 1972.

[2] H. AlZER, On some inequalities for the gamma and psi functions, Math. Comput. 66 (1997), 373-389.

[3] N. BATIR, Inequalities for the gamma function, Arch. Math. (Basel) 91 (2008), 554-563.

[4] N. BATIR, Very accurate approximations for the factorial function, J. Math. Inequal. 4 (2010), 335344.

[5] J. Bukac, T. Burić And N. Elezović, Stirling's formula revisited via some classical and new inequalities, Math. Inequal. Appl. 14 (2011), 235-245.

[6] S. R. Bulò, E. R. HancockB, F. AzizB And M. Pelilloa, Efficient computation of Ihara coefficients using the Bell polynomial recursion, Linear Algebra Appl. 436 (2012), 1436-1441.

[7] T. BURIĆ AND N. ElEzović, Bernoulli polynomials and asymptotic expansions of the quotient of gamma functions, J. Comput. Appl. Math. 235 (2011), 3315-3331.

[8] T. BURIĆ AND N. ElEzOVIĆ, New asymptotic expansions of the quotient of gamma functions, Integral Transforms Spec. Funct. 23 (2012), 355-368.

[9] T. BURIĆ AND N. ElEZOVIĆ, New asymptotic expansions of the gamma function and improvements of Stirling's type formulas, J. Comput. Anal. Appl. 13 (2011), 785-795.

[10] C. A. Charalambides, Enumerative Combinatorics, in: CRC Press Series on DiscreteMathematics and its Applications, Chapman \& Hall/CRC, Boca Raton, FL, 2002.

[11] C.-P. CHEN, Unified treatment of several asymptotic formulas for the gamma function, Numer. Algor. 64 (2013), 311-319.

[12] C.-P. Chen AND L. Lin, Remarks on asymptotic expansions for the gamma function, Appl. Math. Lett. 25 (2012), 2322-2326.

[13] L. Comtet, Advanced Combinatorics, D. Reidel Publishing Co., Dordrecht, 1974.

[14] E. T. Copson, Asymptotic Expansions, Cambridge University Press, 1965.

[15] D. CviJović, New identities for the partial Bell polynomials, Appl. Math. Lett. 24 (2011), $1544-1547$.

[16] R. W. Gosper, Decision procedure for indefinite hypergeometric summation, Proc. Natl. Acad. Sci. USA 75 (1978) 40-42.

[17] H. W. Gould, The Girard-Waring power sum formulas for symmetric functions and Fibonacci sequences, Fibonacci Quart. 37 (1999), 135-140.

[18] E. A. KARATSUBA, On the asymptotic representation of the Euler gamma function by Ramanujan, J. Comput. Appl. Math. 135 (2001), 225-240.

[19] P. LusCHNy, Approximation formulas for the factorial function n!, http://www.luschny.de/math/factorial/approx/SimpleCases.html. 
[20] C. Mortici, Sharp inequalities related to Gosper's formula, C. R. Math. Acad. Sci. Paris 348 (2010), $137-140$.

[21] C. Mortici, On Gospers formula for the Gamma function, J. Math. Inequal. 5 (2011), 611-614.

[22] G. Nemes, More accurate approximations for the gamma function, Thai J. Math. 9 (2011), 21-28.

[23] J. Riondan, Combinatorial Identities, Reprint of the 1968 original, Robert E. Krieger Publishing Co., Huntington, NY, 1979. 